\title{
Alterações na composição florística das algas da Praia de Boa Viagem (Niterói, RJ)
}

\author{
ANDRÉ TAOUIL ${ }^{1,3}$ e YOCIE YONESHIGUE-VALENTIN ${ }^{2}$
}

(recebido: 19 de dezembro de 2001; aceito: 5 de junho de 2002)

\begin{abstract}
Changes in the marine flora of the Boa Viagem beach (Niterói, RJ)). During the past three decades, the Guanabara Bay suffered continuous degradation, leading to severe changes in the surrounding area and environmental damages. Besides significant loss of its natural landscape, the quality of its water decreased with continuous disposal of non-treated domestic organic sewage and industrial effluents. The present study aimed to survey the algal flora of Boa Viagem beach (Niterói, RJ) and to compare it with information gathered three decades ago. Results showed a decrease of 30 taxa (49\%) of the algal species and an increase of 14 species $(23 \%)$ in comparison with algal flora recorded three decades ago. The actual algal flora is dominated by Ulva fasciata Delile and Enteromorpha compressa (L.) Nees, species indicative of organic pollution. These results elucidate the environmental impacts caused upon the macroalgae community of the Guanabara Bay during throughout the period.
\end{abstract}

RESUMO - (Alterações na composição florística das algas da Praia de Boa Viagem (Niterói, RJ)). A Baía de Guanabara no decorrer da ocupação de suas margens sofreu uma continua degradação, levando-a a grandes modificações em seu entorno e a inúmeros danos ambientais. Além de uma significativa alteração paisagística, ocorreu também uma queda na qualidade de suas águas, devido ao lançamento de grande quantidade de efluentes não tratados (domésticos e industriais). Este trabalho teve como objetivo inventariar a flora algácea da praia de Boa Viagem (Niterói, RJ), compará-la com levantamentos realizados há três décadas. Os resultados mostraram desaparecimento de 30 táxons (49\%) outrora existentes no início da década de 70 e o aparecimento de 14 espécies (23\%). A flora atual é dominada por Ulva fasciata Delile e Enteromorpha compressa (L.) Nees, espécies potencialmente indicadoras de poluição orgânica. Tais resultados evidenciam que, ao longo dessas três décadas, uma série de impactos trouxe profundas alterações na comunidade de macroalgas.

Key words - Benthos, flora, Guanabara Bay, organic pollution, seaweeds

\section{Introdução}

As comunidades de macroalgas marinhas, por serem compostas de organismos sésseis, sofrem efeitos de diversos elementos do meio circundante, o que as faz excelentes sensores biológicos das condições ambientais e das tendências evolutivas de seus ecossistemas (Borowitzka 1972, Littler \& Murray 1975, Levine 1984, Ducrotoy 1999, Díez et al. 1999).

A eutrofização, corresponde a um complexo processo caracterizado pelo enriquecimento das águas com nutrientes, o qual afeta os organismos de forma diferenciada e pode provocar distúrbios que afetam a diversidade e a distribuição de comunidades (Gorostiaga \& Díez 1996). Em geral, o ambiente responde com alterações e, freqüentemente,

1. Universidade Estadual do Norte Fluminense, CBB, LCA. Av. Alberto Lamego 2000, 28015-620 Campos dos Goytacazes, RJ Brasil.

2. Universidade Federal do Rio de Janeiro, CCS, Instituto de Biologia, Departamento de Botânica. Bloco A, Cidade Universitária, Ilha do Fundão, 21941-590 Rio de Janeiro, RJ Brasil.

3._Autor para correspondência: ataouil@uenf.br simplificação da estrutura das comunidades (Díez et al. 1999, Leaf \& Chatterjee 1999).

A Baía de Guanabara vem sofrendo, ao longo das últimas décadas, progressivo comprometimento ambiental em decorrência de despejos domésticos e industriais não tratados. Este estudo teve como objetivo avaliar os efeitos de alterações da diversidade algácea na praia de Boa Viagem, Município de Niterói, RJ. Esta avaliação compreendeu um inventário florístico e sua comparação com levantamentos realizados há 30 anos por Yoneshigue-Braga (1970a, b, 1971, 1972a, b). Estes resultados servirão também para se verificar, no futuro, os efeitos do Programa de Despoluição da Baía de Guanabara.

\section{Material e métodos}

Área de estudo - A Baía de Guanabara tem $377 \mathrm{~km}^{2}$, com perímetro de $131 \mathrm{~km}$. A sua profundidade média atual é de 7,6 m, com volume d'água de $3,058 \times 10^{9} \mathrm{~m}^{3}$ (Amador 1997).

A carga de efluentes despejada diariamente na Baía de Guanabara corresponde a 465 toneladas de esgotos domésticos, sendo que apenas 68 toneladas são tratadas. A carga orgânica é de 400 toneladas, das quais 64 toneladas 
correspondem a carga industrial. A carga de metais pesados é de 0,3 toneladas, a carga de óleo é de 7 toneladas e a carga orgânica de lixo doméstico de 6 toneladas (Teixeira et al. 1987, Mayr et al. 1989, Lavrado et al. 1991, SOSP 1992).

A praia de Boa Viagem (figura 1) pode ser classificada como moderadamente batida, tendo como característica principal ser formada por costões rochosos e pequenos blocos de rocha (matacões), entremeados por areia. A salinidade varia entre 32,1 e $36,9 \%$, a temperatura oscila entre 22,4 e $24,9^{\circ} \mathrm{C}$ e a transparência da água obtida por disco de Secchi entre 2,3 e $2,7 \mathrm{~m}$, com concentrações de fósforo total entre 1,93 a $4,84 \mu \mathrm{M}$ e de clorofila $a$ entre 4,0 e $28 \mu \mathrm{g} .1^{-1}$ (Mayr et al. 1989, Paranhos et al. 2001). Araújo (1989) registrou, para esta praia, concentrações de fosfato de $1,26 \pm 0,53 \mu \mathrm{M}$, de nitrito de 0,97 $\pm 0,63 \mu \mathrm{M}$ e de amônia de 11,62 \pm 8,69 $\mu \mathrm{M}$.

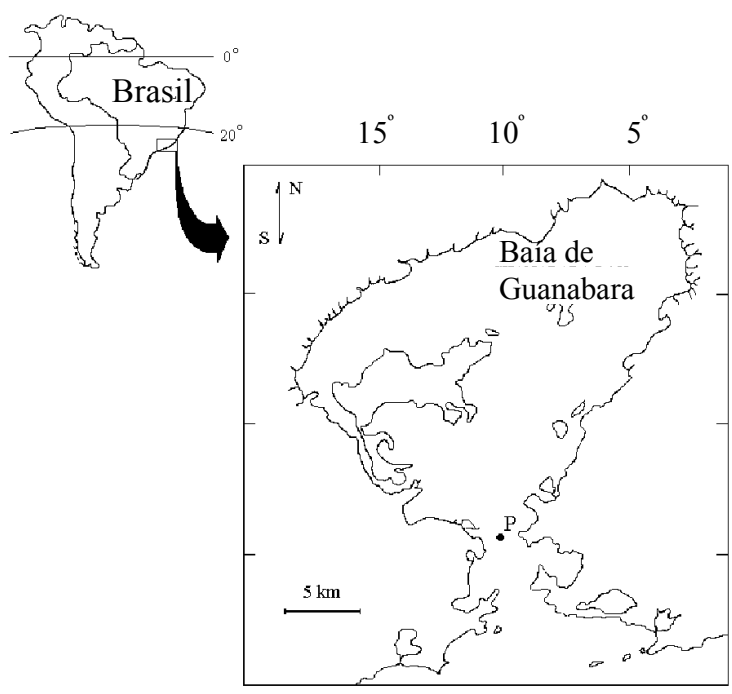

$43^{\circ} \mathrm{W}$

$45^{\circ}$

$50^{\circ}$

$55^{\circ}$

$23^{\circ} \mathrm{S}$

Figura 1. Localização da praia de Boa Viagem (P) na Baía de Guanabara, estado do Rio de Janeiro.

A escolha do local de coleta deu-se em função dos seguintes fatores: (i) existência de informações pretéritas acerca da distribuição da flora algácea local (YoneshigueBraga 1970a, b, 1971, 1972a, b, Mitchell et al. 1979, Teixeira et al. 1987), bem como estudos sobre aspectos ecológicos envolvendo a comunidade de macroalgas desta praia (Teixeira et al. 1984a, b, Carneiro et al. 1987) e (ii) a identificação da praia de Boa Viagem como moderadamente impactada de acordo com Teixeira et al. (1984a, 1987) e Morand \& Briand (1996).

Coleta das amostras - Foram efetuadas quatro coletas sazonais arbitrárias (outubro de 1996, fevereiro de 1997, maio de 1997 e agosto de 1997) das macroalgas nos costões rochosos e matacões da praia de Boa Viagem, sempre em marés baixas. Os espécimens algáceos foram retirados do substrato através de espátula de pedreiro, raspando-o integralmente a fim de serem obtidas plantas inteiras sem prejuízo de suas bases (Yoneshigue-Braga 1970a, b, 1971, 1972a, b). Essas amostras foram imediatamente fixadas numa solução de formol comercial a $4 \%$ preparada em água do mar tamponada com bórax, sendo em seguida triadas em laboratório.

A identificação taxonômica seguiu as técnicas clássicas usadas em Ficologia (Joly 1965, Yoneshigue-Braga 1970a, b, 1971, 1972a, b, Mitchell et al. 1979, Yoneshigue 1985, Amado-Filho 1991 e Wynne 1998). Foram considerados, portanto, os caracteres que compõem a morfologia externa (hábito geral, tipo de fixação, organização do talo e presença de órgãos de reprodução) e a morfologia interna (tipos de células corticais, medulares e outras estruturas quando presentes). Os cortes transversais das frondes foram efetuados à mão livre por meio de uma lâmina de barbear. As medidas das estruturas foram tomadas em pelo menos cinco exemplares de cada espécie.

\section{Resultados}

Foram encontrados, no total, 45 táxons (tabela 1), sendo uma Phaeophyta (2\%); 16 Chlorophyta (36\%) e 28 Rhodophyta (62\%). Na primavera foram encontradas 31 espécies, no verão 25, no outono 32 e no inverno 26 espécies (figura 2, tabela 1) e 17 espécies ocorreram nas quatro estações do ano (tabela 1). Quatro espécies foram registradas no outono e inverno, três foram observadas no verão e outono, e outras, ainda, estiveram presentes em duas ou três estações do ano (tabela 1).

A fisionomia das comunidades foi dominada por algas verdes com talos foliáceos, filamentosos e cenocíticos. As espécies de algas vermelhas filamentosas (por exemplo, Ceramium spp. e Polysiphonia spp., exceto $C$. clavulatum) e a única alga parda detectada (Feldmannia irregularis), ocorreram de modo epifítico apenas em Codium decorticatum.

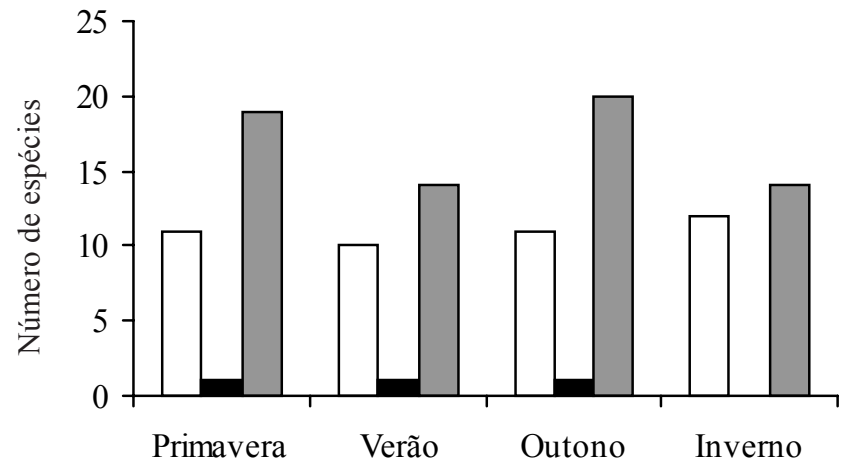

Figura 2. Distribuição sazonal do número de espécies de $\square$ Chlorophyta, $\square$ Phaeophyta e $\square$ Rhodophyta, encontrado na área de estudo. 
Tabela 1. Inventário das espécies ao longo das quatro estações do ano na área estudada. + / - indica a presença ou ausência da espécie na referida estação do ano.

\begin{tabular}{|c|c|c|c|c|}
\hline Espécies & Primavera & Verão & Outono & Inverno \\
\hline \multicolumn{5}{|l|}{ Chlorophyta } \\
\hline Bryopsis plumosa (Huds.) C. Agardh & - & - & + & + \\
\hline Caulerpa fastigiata Montagne & - & + & + & - \\
\hline Chaetomorpha antennina (Bory) Kützing & + & + & + & + \\
\hline Chaetomorpha brachygona Harvey & - & + & - & - \\
\hline Cladophora prolifera (Roth) Kützing & + & + & + & + \\
\hline Cladophora rupestris (L.) Kützing & + & + & + & + \\
\hline Cladophora vagabunda (L.) C. Hoek. & + & + & + & + \\
\hline Codium decorticatum (Woodw.) M. Howe & + & + & + & + \\
\hline Codium taylori P.C. Silva & - & - & - & + \\
\hline Derbesia sp. & + & - & - & - \\
\hline Enteromorpha compressa (L.) Nees & + & + & + & + \\
\hline Enteromorpha flexuosa (Wulfen) J. Agardh & - & - & + & + \\
\hline Entocladia viridis Reinke & + & - & - & - \\
\hline Ulva fasciata Delile & + & + & + & + \\
\hline Ulva lactuca $\mathrm{L}$. & + & - & - & + \\
\hline Ulva rigida $\mathrm{C}$. Agardh & + & + & + & + \\
\hline \multicolumn{5}{|l|}{ Phaeophyta } \\
\hline Feldmannia irregularis (Kützing) Hamel & + & + & + & - \\
\hline \multicolumn{5}{|l|}{ Rhodophyta } \\
\hline Acrochaetium hypneae (Börgesen) \& F. Magne & + & - & - & - \\
\hline Acrochaetium microscopicum (Nägeli ex Kützing) Nägeli & + & - & - & - \\
\hline Amphiroa beauvoisii J.V. Lamouroux & - & + & + & - \\
\hline Amphiroa brasiliana Decne & - & - & + & - \\
\hline Bryocladia thyrsigera (J. Agardh) F. Schmitz & + & - & + & + \\
\hline Centroceras clavulatum (C. Agardh) Mont. & + & + & + & + \\
\hline Ceramium brasiliense A.B. Joly & + & + & + & + \\
\hline Ceramium brevizonatum H.E. Petersen & - & + & - & - \\
\hline Ceramium comptum Börgesen & + & - & - & - \\
\hline Ceramium deslonchampii Chauv ex Duby & + & - & - & - \\
\hline Ceramium flaccidum (Kützing) Ardiss & + & - & - & - \\
\hline Champia salicornioides Harvey & + & + & + & + \\
\hline Chondracanthus acicularis (Röth) Fredericq & + & - & + & - \\
\hline Chondracanthus teedii (Mertens ex Roth) Fredericq & + & + & + & + \\
\hline Chondria atropurpurea Harvey & - & + & + & + \\
\hline Dasya brasiliensis Oliveira Filho \& Yoneshigue-Braga & - & - & + & + \\
\hline Gastroclonium parvum (Hollenberg) C.F. Chang \& B.M. Xia & - & - & + & + \\
\hline Gelidiella pannosa (Feldmann) Feldmann \& Hamel & - & - & - & + \\
\hline Gelidium floridanum W.R. Taylor & - & - & + & - \\
\hline Gelidium pusillum (Stackhouse) Le Jolis & + & + & + & - \\
\hline Grateloupia doryphora (Montagne) M. Howe & + & + & + & + \\
\hline Gymnogongrus griffthsiae (Turner) Mart. & + & + & + & + \\
\hline Herposiphonia bipinnata $\mathrm{M}$. Howe & + & - & - & - \\
\hline Hildenbrandia rubra (Sommerf.) Menegh. & - & + & + & - \\
\hline Jania adhaerens J.V. Lamouroux & + & + & + & + \\
\hline Polysiphonia ferulacea Suhr ex J. Agardh & + & + & + & + \\
\hline Polysiphonia howeii Hollenberg & + & + & + & + \\
\hline Sahlingia subintegra (Rosenvinge) Kormann & + & - & - & - \\
\hline
\end{tabular}




\section{Discussão}

Comparando-se o número total de espécies encontradas na praia de Boa Viagem, por YoneshigueBraga (1970a, b, 1971, 1972a, b) há quase trinta anos e o presente trabalho, constatou-se o desaparecimento de 30 táxons na área de estudo. As espécies que mais representam esta redução pertencem a Chlorophyta, com 4 táxons, Phaeophyta, com dez táxons a menos e Rhodophyta, com decréscimo de dezesseis táxons.

Comparações com levantamentos realizados por Yoneshigue-Braga (1970a, b, 1971, 1972a, b), que encontrou 62 taxa repartidos entre 18 Chlorophyta (29\%), 11 Phaeophyta (18\%) e 33 Rhodophyta (53\%) mostram significativa redução do número de espécies (tabela 2). Comparações com outros trabalhos como os de Mitchell et al. (1979), Teixeira et al. (1984a, 1987) e Carneiro et al. (1987), que não tiveram o levantamento florístico como meta principal, podem levar a superestimativa dos dados, em relação aos de Yoneshigue-Braga (1970a, b; 1971; 1972a, b), que visaram especificamente o levantamento da macroflora de algas da Baía de Guanabara.

A praia de Boa Viagem apresentou, visualmente, uma maciça predominância de espécies de Chlorophyta. Nossos dados mostraram redução no número de espécies da flora local e o desaparecimento progressivo das algas pardas e vermelhas ao longo de três décadas (Carneiro et al. 1987, Teixeira et al. 1987). Resultados semelhantes foram encontrados por Borowitzka (1972), Edwards (1972), Oliveira-Filho \& Berchez (1978), Munda (1980, 1996), Hardy et al. (1993) e Bellgrove et al. (1997).

O desaparecimento de espécies, sobretudo as Phaeophyta de grande e pequeno porte, tais como Sargassum spp., Padina spp., Spatoglossum schroederi,

Tabela 2. Comparação da composição florística atual da praia de Boa Viagem, com levantamentos feitos por outros autores. + / - indica, respectivamente, a presença ou ausência da espécie no referido trabalho.

\begin{tabular}{|c|c|c|c|c|}
\hline \multirow[b]{2}{*}{ Espécies } & \multicolumn{4}{|c|}{ Autores } \\
\hline & $\begin{array}{c}\text { Yoneshigue-Braga } \\
\text { 1970a, b, } 1971 \text { e 1972a, b }\end{array}$ & $\begin{array}{l}\text { Mitchell et al. } \\
1979\end{array}$ & $\begin{array}{c}\text { Teixeira et al. } \\
1987\end{array}$ & Presente estudo \\
\hline \multicolumn{5}{|l|}{ Chlorophyta } \\
\hline Bryopsis corymbosa J. Agardh & + & - & - & - \\
\hline Bryopsis pennata J.V. Lamouroux & + & - & - & - \\
\hline Bryopsis plumosa (Huds.) C. Agardh & + & - & - & + \\
\hline Caulerpa fastigiata Montagne & + & - & - & + \\
\hline Caulerpa racemosa (Forskaal) J. Agardh & + & - & - & - \\
\hline Chaetomorpha antennina (Bory) Kützing & + & + & - & + \\
\hline Chaetomorpha brachygona Harvey & - & - & - & + \\
\hline Cladophora prolifera (Roth) Kützing & + & - & - & + \\
\hline Cladophora rupestris (L.) Kützing & + & - & - & + \\
\hline Cladophora vagabunda (L.) C. Hoek & + & - & + & + \\
\hline Codium decorticatum (Woodw.) M. Howe & + & + & + & + \\
\hline Codium isthmocladum Vickers & - & + & - & - \\
\hline Codium taylori P.C. Silva & + & - & - & + \\
\hline Derbesia marina (Lyngbye) Kjellman & + & - & + & - \\
\hline Derbesia sp. & - & - & - & + \\
\hline Enteromorha flexuosa (Wulfen) J. Agardh & - & - & - & + \\
\hline Enteromorpha compressa (L.) Nees & - & - & + & + \\
\hline Enteromorpha linza (L.) J. Agardh & + & - & - & - \\
\hline Entocladia viridis Reinke & + & - & - & + \\
\hline Ulva fasciata Delile & + & + & + & + \\
\hline Ulva lactuca $\mathrm{L}$. & + & - & - & + \\
\hline Ulva rigida $\mathrm{C}$. Agardh & - & - & - & + \\
\hline \multicolumn{5}{|l|}{ Phaeophyta } \\
\hline Bachelotia antillarum (Grunow) Gerloff & + & - & - & - \\
\hline Colpomenia sinnиоsa (Roth) Dèrbes \& Solier & + & - & + & - \\
\hline
\end{tabular}


(cont.)

\begin{tabular}{lcccc}
\hline & \multicolumn{3}{c}{ Autores } \\
\cline { 2 - 4 } Espécies & Yoneshigue-Braga & Mitchell et al. & Teixeira et al. & Presente estudo \\
& $1970 \mathrm{a}, \mathrm{b}, 1971 \mathrm{e} 1972 \mathrm{a}, \mathrm{b}$ & 1979 & 1987 & \\
\hline
\end{tabular}

Phaeophyta

Feldmannia irregularis (Kützing) Hamel

Padina gymnospora (Kützing) Sond.

Padina vickersiae Hoyt

Ralfsia expansa (J. Agardh) J. Agardh

Rosenvingea sanctae-crucis Börgesen

Sargassum cymosum C. Agardh

Sargassum cymosum var. stenophyllum (Mertens) Grow

Sargassum sp.

Sargassum vulgare C. Agardh

Spatoglossum schroederi (C. Agardh) Kützing

Rhodophyta

Acrochaetium hypneae Böergesen \& F. Magne

Acrochaetium microscopicum (Nägeli ex Kützing)

Nägeli

Aglaothamnion cordatum (Böergesen)

Feldmann-Mazoyer

Amphiroa beauvoisii J.V. Lamouroux

Amphiroa brasiliana Decne

Arthrocardia flabellata (Kützing) Manza.

Bostrychia tenella f. terrestre (J.V. Lamouroux)

J. Agardh

Bostrychia tenella J.V. Lamouroux

Bryocladia thyrsigera (J. Agardh) F. Schmitz

Centroceras clavulatum (C. Agardh) Mont.

Ceramium brasiliense A.B. Joly

Ceramium brevizonatum H.E. Petersen

Ceramium comptum Börgesen

Ceramium dawsoni A.B. Joly

Ceramium deslongchampii Chauvy ex Duby

Ceramium flaccidum (Kützing) Ardiss

Champia salicornioides Harvey

Chondracanthus acicularis (Roth) Fredericq

Chondracanthus teedei (Mertens ex Roth) Fredericq

Chondria atropurpurea Harvey

Dasya brasiliensis Oliveira Filho \& Yoneshigue-Braga

Gastroclonium parvum (Hollenberg)

C.F. Chang \& B.M. Xia

Gelidiella pannosa (Feldmann) Feldmann \& Hamel

Gelidiela sp.

Gelidium floridanum W.R. Taylor

Gelidium pusillum (Stackhouse) Le Jolis

Gracilaria cervicornis (Turner) J.Agardh

Gracilaria lacinulata (H. West) M. Howe

Gracilaria lacinulata (H. West in Vahl) M. Howe

Grateloupia doryphora (Montagne) M. Howe

Grateloupia filicina (J.V. Lamouroux) C. Agardh

Gymnogongrus griffithsiae (Turner) Mart.

Gymnothamnion elegans

(Schousb. ex C. Agardh) J. Agardh 
(cont.)

\begin{tabular}{|c|c|c|c|c|}
\hline \multirow[b]{2}{*}{ Espécies } & \multicolumn{4}{|c|}{ Autores } \\
\hline & $\begin{array}{l}\text { Yoneshigue-Braga } \\
\text { 970a, b, } 1971 \text { e 1972a, b }\end{array}$ & $\begin{array}{l}\text { Mitchell et al. } \\
1979\end{array}$ & $\begin{array}{c}\text { Teixeira et al. } \\
1987\end{array}$ & Presente estudo \\
\hline \multicolumn{5}{|l|}{ Rhodophyta } \\
\hline Herposiphonia bipinnata M. Howe & - & - & - & + \\
\hline Herposiphonia secunda (C. Agardh) Ambronn & + & - & - & - \\
\hline Hildenbrandtia rubra (Sommerf.) Menegh. & + & - & - & + \\
\hline Hypnea musciformis (Wulfen) J.V. Lamouroux & + & - & - & - \\
\hline Hypnea spinella (C. Agardh) Kützing & - & - & + & - \\
\hline Jania adhaerens J.V. Lamouroux & + & - & - & + \\
\hline Polysiphonia ferulacea Suhr ex J. Agardh & + & - & - & + \\
\hline Polysiphonia howei Hollenberg & - & - & - & + \\
\hline Polysiphonia spp. & - & - & + & - \\
\hline Polysiphonia subtilissima Montagne & + & - & + & - \\
\hline $\begin{array}{l}\text { Porphyra acantophora var. acantophora } \\
\text { E.C. Oliveira \& J. Coll. }\end{array}$ & + & - & - & - \\
\hline Rhodothamniella codicola (Börgesen) Bidoux & + & - & - & - \\
\hline Rhodymenia pseudopalmata (Lamouroux) Silva & lva & - & - & - \\
\hline Sahlingia subintegra (Rosenvinge) Kormann & + & - & - & + \\
\hline Scinaia complanata (Collins) Cotton & + & - & - & - \\
\hline Spermothamnion nonatoi A.B. Joly & + & - & - & - \\
\hline Stylonema alsidii (Zanardini) K.M. Drew & + & - & - & - \\
\hline
\end{tabular}

Giffordia spp. e Bachelotia antillarum, pode estar associado à toxicidade de compostos orgânicos antropogênicos presentes nos esgotos doméstico e industrial não tratados e à sua interferência nos ciclos de vida das algas pardas (Jaenicke 1977, Teixeira et al. 1987, Bellgrove et al. 1997). Por outro lado, a presença dos gêneros Codium, Gelidium, Gelidiella, Gymnogongrus e Amphiroa entre outros, é característica de ambientes moderadamente impactados (Teixeira et al. 1987, Gorostiaga \& Díez 1996). Essas espécies podem ser utilizadas como bioindicadoras em programas de despoluição e indicam o nível de degradação da área impactada (Munda 1980, 1996, Mitchell et al. 1990).

Uma informação importante foi o registro de 14 novas ocorrências semelhante ao registrado por Oliveira-Filho \& Berchez (1978) para a baía de Santos. Gelidium pusillum, Gastroclonium parvum e Polysiphonia howeii embora não citadas por Yoneshigue-Braga (1970a, b, 1971, 1972a, b) foram observadas por Teixeira et al. (1984a, 1987) e Carneiro et al. (1987) não constituindo-se em novas ocorrências para esta praia.

O incremento da eutrofização ao longo de trinta anos perturbou com decréscimo a diversidade e alterou a composição específica das macroalgas marinhas no ecossistema estuarino da baía de Guanabara, como caracterizado na área de estudo. Ressalta-se a necessidade do contínuo acompanhamento na composição específica das macroalgas levando-se também em consideração a estrutura de suas comunidades.

Agradecimentos - Este trabalho foi realizado sob os auspícios do Conselho Nacional de Pesquisa e Desenvolvimento Científico (CNPq, Proc. AI 521783/96-0), do Fundo Mundial para a Natureza no Brasil (WWF CSR 101-98) e do Pronex 661421/1996-1 aos quais os autores expressam seus agradecimentos.

\section{Referências bibliográficas}

AMADO-FILHO, G.M. 1991. Algas marinhas bentônicas do litoral de Saquarema a Itacoatiara (RJ). Dissertação de mestrado, Universidade Federal do Rio de Janeiro, Rio de Janeiro.

AMADOR, E.S. 1997. Baía de Guanabara e ecossistemas periféricos: Homem e natureza. Reproarte Gráfica e Editora Ltda, Rio de Janeiro. 
ARAÚJO, F.V. 1989. Qualidade microbiana de águas litorâneas destinadas a balneabilidade e a extração de moluscos, no Rio de Janeiro e Niterói. Monografia, Universidade Federal do Rio de Janeiro, Rio de Janeiro.

BELLGROVE, A., CLAYTON, M.N. \& QUINN, G.P. 1997. Effects of secondarily treated sewage effluent on intertidal macroalgal recruitment processes. Marine \& Freshwater Research 48:137-146.

BOROWITZKA, M.A. 1972. Intertidal algal species diversity and the effect of pollution. Australian Journal of Marine Freshwater Research 23:73-84.

CARNEIRO, M.E.R., MARQUES, A.N., PEREIRA, R.C., CABRAL, M.M.O. \& TEIXEIRA, V.L. 1987. Estudos populacionais de Ulva fasciata Delile, indicadora de poluição na Baía de Guanabara. Nerítica 2 (supl.):201-212.

DÍEZ, I., SECILLA, A., SANTOLARIA, A. \& GOROSTIAGA, J.M. 1999. Phytobenthic intertidal community structure along an environmental pollution gradient. Marine Pollution Bulletin 38:463-472.

DUCROTOY, J.P. 1999. Indications of change in the marine flora of the North Sea in the 1990s. Marine Pollution Bulletin 38:646-654.

EDWARDS, P. 1972. Benthic algae in polluted estuaries. Marine Pollution Bulletin 3:55-60.

GOROSTIAGA, J.M. \& DÍEZ, I. 1996. Changes in the sublittoral benthic marine macroalgae in the polluted area of Abra de Bilbao and proximal coast (Northern Spain). Marine Ecology Progress Series 130:157-167.

HARDY, F.G., EVANS, S.M. \& TREMAYNE, M.A. 1993. Long-term changes in the marine macroalgae of three polluted estuaries in north-east England. Journal of Experimental Marine Biology \& Ecology 172:81-92.

JAENICKE, L. 1977. Sex hormones of brown algae. Naturwissenschaften 64:69-75.

JOLY, A.B. 1965. Flora marinha do litoral norte do estado de São Paulo e regiões circunvizinhas. Boletim da Faculdade de Filosofia, Ciências e Letras da Universidade de São Paulo, (Série Botânica 21) 294:1-393.

LAVRADO, H., MAYR, L.M., CARVALHO, V. \& PARANHOS, R. 1991. Evolution (1980-1990) of ammonia and dissolved oxygen in Guanabara Bay, RJ, Brazil. In Proceedings of $7^{\text {th }}$ Symposium of Coastal and Ocean Management - Coastal Zone 91 (O.T. Magon, H. Convex, V. Tippie, L.T. Tobin \& D. Clarke, eds.). p.3234-3245.

LEAF, S.S. \& CHATTERJEE, R. 1999. Developing a strategy on eutrophication. Water Science and Technology 39:307-314.

LEVINE, H.G. 1984. The use of seaweeds for monitoring coastal waters. In Algae as ecological indicators (L.E. Shubert, ed.). Academic Press, London.

LITTLER, M.M. \& MURRAY, S.N. 1975. Impact of sewage on the distribution, abundance and community structure of rocky intertidal macro-organisms. Marine Biology 30:277-291.
MAYR, L.M., TENENBAUN, D.R., VILLAC, M.C., PARANHOS, R., NOGUEIRA, C.R., BONECKER, S.L.C. \& BONECKER, A.C. 1989. Hydrobiological characterization of Guanabara Bay. In Coastlines of Brazil (O.T. Maggon \& C. Neves, eds.). American Society of Civil Engineers, New York, p.124-139.

MITCHELL, G.J.P., SZÉCHY, M.T.M. \& MITSUYA, L.A. 1979. Sinopse das clorofíceaes marinhas bentônicas do litoral do estado do Rio de Janeiro. Leandra 8-9:91-123.

MITCHELL, G.J.P., NASSAR, C.A.G., MAURAT, M.C.S. \& FALCÃO, C. 1990. Tipos de vegetação marinha da baía do Espírito Santo sob a influência da poluição Espírito Santo (Brasil). In Anais do II Simpósio de Ecossistemas da Costa Sul e Sudeste Brasileira Estrutura, Função e Manejo (S. Watanabe, ed.). Aciesp, São Paulo, p.202-214.

MORAND, P. \& BRIAND, X. 1996. Excessive growth of macroalgae: a symptom of environmental disturbance. Botanica Marina 39:491-516.

MUNDA, I.M. 1980. Changes in the benthic algal associations of the vicinity of Rovinj (Istrian coast, north Adriatic) caused by organic wastes. Acta Adriatica 21:299-332.

MUNDA, I.M. 1996. The northern Adriatic Sea. In Marine benthic vegetation. Recent changes and the effects of eutrophication (W. Schramm \& P.H. Nienhuis, eds.). Springer-Verlag, Berlin, p.369-402.

OLIVEIRA-FILHO, E.C.de \& BERCHEZ, F.A.S. 1978. Algas marinhas bentônicas da Baía de Santos Alterações da flora no período de 1957-1978. Boletim de Botânica da Universidade de São Paulo, 6:49-59.

PARANHOS, R., ANDRADE, L., MENDONÇA-HAGLER, L.C. \& PFEIFFER, W.C. 2001. Coupling bacterial abundance with production in a polluted tropical coastal bay. In Aquatic microbial ecology in Brazil (B.M. Faria, V.F. Farjalla \& F.A. Esteves, eds.). Series Oecologia Brasiliensis, PPGE-UFRJ, Rio de Janeiro, v.9: p.117-132.

SOSP, 1992. Secretaria de Estado de Obras e Serviços Públicos. Governo do Estado do Rio de Janeiro. Divulgação das obras de despoluição da Baía de Guanabara.

TEIXEIRA, V.L., ALMEIDA S.A.S., FLEURY, B.G., LINS, P.C.M. \& BENFICA, R.C. 1984a. Comunidades de algas bentônicas e fauna acompanhante de poças de maré de Niterói (RJ). In Anais IV Congresso da Sociedade Botânica de São Paulo (T.S. Meulheim, ed.). Sociedade Botânica de São Paulo, Taubaté, p.93-97.

TEIXEIRA, V.L., PEREIRA, R.C., ALMEIDA S.A.S. \& JÚNIOR, A.N.M. 1984b. Estudo comparativo de populações de Codium decorticatum (Woodward) Howe em Niterói (RJ), em diferentes condições ambientais. In Anais IV Congresso da Sociedade Botânica de São Paulo (T.S. Meulheim, ed.). Sociedade Botânica de São Paulo, Taubaté, p.87-91. 
TEIXEIRA, V.L., PEREIRA, R.C., JÚNIOR, A.N.M., LEITÃO FILHO, C.M. \& SILVA, C.A.R. 1987. Seasonal variations in infralitoral seaweed communities under a pollution gradient in Baía de Guanabara, Rio de Janeiro (Brazil). Ciência e Cultura 39:423-428.

WYNNE, M.J. 1998. A checklist of benthic marine algae of the tropical and subtropical western Atlantic: first revision. Nova Hedwigia Beiheft 116:1-155.

YONESHIGUE-BRAGA,Y. 1970a. Flora marinha bentônica da Baía de Guanabara e cercanias. I. - Chlorophyta. Instituto de Pesquisas da Marinha, Rio de Janeiro, Publicação 42, p.1-55.

YONESHIGUE-BRAGA,Y. 1970b. Flora marinha bentônica da Baía de Guanabara e cercanias. II. - Phaeophyta. Instituto de Pesquisas da Marinha, Rio de Janeiro, Publicação 45, p.1-31.
YONESHIGUE-BRAGA,Y. 1971. Flora marinha bentônica da Baía de Guanabara e cercanias. III. - Rhodophyta. 1. Goniotrichales, Bangiales, Compsopogonales, Nemalionales e Gelidiales. Instituto de Pesquisas da Marinha, Rio de Janeiro, Publicação 55, p.1-36.

YONESHIGUE-BRAGA,Y. 1972a. Flora marinha bentônica da Baía de Guanabara e cercanias. III. - Rhodophyta. 2. Cryptonemiales, Gigartinales e Rhodymeniales. Instituto de Pesquisas da Marinha, Rio de Janeiro, Publicação 62, p.1-39.

YONESHIGUE-BRAGA,Y. 1972b. Flora marinha bentônica da Baía de Guanabara e cercanias. III. - Rhodophyta. 3. Ceramiales. Instituto de Pesquisas da Marinha, Rio de Janeiro, Publicação 65, p.1-49.

YONESHIGUE, Y. 1985. Taxonomie et écologie des algues marines dans la région de Cabo Frio (Rio de Janeiro, Brésil). Doucteur de d'Etat-Sciences, Université d'AixMarseille, Marseille. 